\title{
La réintroduction du français en Colombie, un défi qui nous concerne en tant qu'enseignants
}

\author{
La reintroducción del francés en Colombia, \\ un desafío que concierne a todos
}

\author{
The re-introduction of French in Colombia, \\ a challenge which concerns us all
}

Fanny Baquero ${ }^{1}$

Adriana Silva ${ }^{2}$

Résumé.

La réintroduction du français en Colombie est un projet qui existe depuis 2008, ce projet est important car il cherche à développer une éducation bilingue de qualité dans les écoles publiques. Le but de cet article est de montrer l'évolution de l'enseignement du français de l'école La Candelaria et les perceptions de tous les acteurs impliqués dans ce projet éducatif de bilinguisme. Après avoir réalisé une étude de cas nous trouvons que l'école La Candelaria présente plusieurs contextes qui montrent une certaine fragilité dans le processus de formation. Néanmoins, nous trouvons que la langue française a une fonction sociale dans l'école et elle offre la possibilité d'être utilisée comme un outil éducatif. Cette école est susceptible de changements qui pourraient améliorer l'éducation à travers la langue française et fournir aux élèves des possibilités pédagogiques afin de réussir leur formation et d'obtenir ainsi une meilleure qualité éducative.

Mots clés

Français langue étrangère, éducation, bilinguisme.

Resumen

La reintroducción del francés en Colombia es un proyecto que existe desde 2008. Este proyecto es importante pues busca desarrollar una educación bilingüe de calidad en los colegios públicos. El objetivo de este artículo es mostrar la evolución de la enseñanza del francés en el colegio La Candelaria en Bogotá y las percepciones de todos los actores implicados en este proceso educativo. Después de haber realizado un estudio de caso encontramos que el colegio La Candelaria presenta múltiples contextos con un alto índice de vulnerabilidad en el proceso de formación. Sin embargo, también encontramos que la lengua francesa tiene una función social en el colegio y ésta ofrece la posibilidad de ser utilizada como una herramienta educativa. Este colegio es susceptible de cambios que podrían mejorar el proceso de formación a través de la lengua francesa y se podría brindar a los alumnos un escenario pedagógico diferente para llevar a cabo una formación de calidad.

\section{Palabras clave}

Francés lengua extranjera, educación, bilingüismo.

1 Pontificia Universidad Javeriana, Bogotá, Colombia. baquerof@javeriana.edu.co. fjbaqueroc@unal.edu.co

2 Pontificia Universidad Javeriana, Bogotá, Colombia. silva.adriana@javeriana.edu.co 
Universidad Pedagógica Nacional

Facultad de Humanidades

\section{Abstract}

The re-introduction of French in Colombia is a project which has been in existence since 2008. This project is important because it seeks to develop quality bilingual education in public secondary schools. The aim of this article is to show the evolution of French teaching in 'La Candelaria' school in Bogota and the perceptions of everyone involved in this educational process. After carrying out a case study, we find that there are various contexts in La Candelaria school which have a high degree of vulnerability in the educational process. Nevertheless, we also find that the French language has a social function in the school and it is therefore possible to use it as an educational tool. This school is susceptible to changes which could improve the educational process by means of the French language and it could provide the students with a different pedagogical setting in order to implement quality education.

\section{Keywords}

French as a foreign language, education, bilingualism.

Artículo recibido el 1 de octubre de 2012 y aprobado el 7 de febrero de 2014

"Le premier sentiment d'un enfant est de s'aimer lui-même; et le second, qui dérive du premier, est d'aimer ceux qui l'approchent; car, dans l'état de faiblesse où il est, il ne connaît personne que par l'assistance et les soins qu'il reçoit 》.

Rousseau. J.J Emile ou de l'éducation. Livre IV

L'enseignement des langues [...] comporte à la fois une finalité interne, c'est-à-dire une visée pratique et opérationnelle [...] et une finalité externe, c'est-à-dire une visée sociale et culturelle.

\section{Claude Springer}

Nous allons aborder dans notre étude de cas la réintroduction du français en Colombie, car ce phénomène est devenu à notre égard très important. Notre analyse est centrée sur l'école publique « La Candelaria », puisqu'il s'agit de l'institution pionnière de la convention entre le gouvernement colombien et le gouvernement français.

A partir de la recherche faite, dans une première partie nous faisons une réflexion sur l'importance de léducation pour tous. Dans un deuxième moment, nous présentons le noyau principal de cet article, à savoir, la réintroduction du français en Colombie, et nous citons les institutions impliquées dans ce projet. Puis nous exposons la démarche du projet du bilinguisme de la réintroduction du français en
Colombie au sein de l'école La Candelaria et, en plus, nous montrons les avis des acteurs du processus denseignement/apprentissage. Finalement, nous présentons les conclusions obtenues dans cette étude.

\section{Projet de bilinguisme "La réintroduction du français "}

Depuis quelques années la réintroduction du français réapparait. En effet, la tendance aujourd'hui est d'avoir la connaissance de plusieurs langues, de soutenir encore le développement humain dans tous les aspects de la vie et de contribuer à l'évolution économique, sociale et culturelle. Les langues deviennent donc une prise substantielle et le français fait notamment partie de ces acquis.

Nous estimons que le capitalisme globalisé d'aujourd'hui provoque une désintégration du droit fondamental de l'éducation. De nos jours toute restructuration consent de nouvelles fonctions dans la société comme par exemple : édifier une éducation qui cherche absolument la compétitivité ayant pour objectif la recherche d'emploi, en oubliant que l'éducation de l'homme et du citoyen a une place au sein de la société qui cherche à faire de léducation un moyen essentiel pour aider le développement de l'esprit- Aujourd'hui, lidée ne vise l'éducation que comme un outil favorisant le travail. 
Le droit à l'éducation figure parmi les droits fondamentaux de l'être humain. Il est aussi considéré comme la clef de l'exercice des autres droits liés à la personne humaine. C'est un droit qui contribue d'une manière très décisive dans l'autonomisation de l'individu. Il constitue à cet égard un outil principal pour la personne humaine, notamment pour les personnes économiquement et socialement marginalisées, de sortir de la misère et de la pauvreté. En plus, la jouissance des autres droits humains préconise ce qu'on appelle 'l'éducation aux droits de l'homme $\gg{ }^{3}$

Cette citation dénote en quelque sorte la fonction de l'éducation et son bouleversement provoqué par la globalisation, nous conduisant par conséquent à une réflexion sur notre rôle comme enseignants et surtout comme enseignants de la langue française. Pour cela, nous devons faire de cette langue un instrument important pour que les élèves puissent développer des compétences non seulement pour leur vie professionnelle mais aussi pour leur vie personnelle. L’enseignement de la langue française est un outil qui permet aux élèves d'avoir des connaissances linguistiques et aussi de développer des compétences vers une formation plus intégrale.

Nous voulons aborder maintenant le noyau principal de cet article : La réintroduction du français en Colombie. A cet égard, il est pertinent de dire que depuis quelques années il existe un intérêt réciproque entre le ministère de léducation et les gouvernements français et colombien pour faire que la langue française soit réintroduite dans les écoles publiques. Pour illustrer cet intérêt nous allons nous intéresser à un article intitulé : Étudiants de la ville de Bogotá apprendront le français ${ }^{4}$, lequel révèle cette réalité.

3 Le Conseil Economique et Social (Nations Unies), Comité des droits économiques, sociaux et culturels, 21ème session, 15 octobre- 3 décembre 1999, Rapport annuel sur le droit à l'éducation, p. 1.

4 Document pris, adapté et traduit de : http://www.bogota.gov.co/ portel/libreria/php/x_frame_detalle.php?id=33989. Consulté le 1.03.12
Le secrétariat d'éducation de Bogotá et l'ambassade de France ont signé une convention pour commencer l'opération du programme d'enseignement/ apprentissage du français et d'autres domaines de la connaissance de la langue française dans les écoles publiques. Lécole la Candelaria a été la première institution qui a désigné le français comme langue étrangère. Mais il faut remarquer que le français s'utilise comme outil d'apprentissage dans d'autres cours faisant partie du curriculum.

Partant de cette convention et sous le soutien de l'ambassade et d'autres liens stratégiques avec des institutions d'éducation supérieure et des organismes spécialisés, le secrétariat de l'Education, selon un article publié en 2008, entreprendra des actions afin de/d' :

- Attester des enseignants selon le niveau de langue française, développer et appliquer des processus pédagogiques pour l'enseignement du français dans les écoles publiques de Bogotá.

- Développer des pilotages basés sur léducation bilingue dans les différentes écoles publiques. Ce pilotage prétend d'insuffler le français dans d'autres domaines.

- Intégrer une éducation bilingue dans les écoles secondaires où il existe une éducation spécialisée en langues.

- Introduire les stages pédagogiques dans les différents niveaux scolaires des écoles en s'appuyant sur des universités et des écoles privées spécialisées en langues.

- Adapter les salles pour l'enseignement des langues en se servant des nouvelles technologies de la communication.

D’après ce même article, lobjectif essentiel du Secrétariat d'Education de Bogotá est de contribuer de manière importante au développement des processus éducatifs afin que les étudiants de Bogotá puissent avoir une éducation de qualité et à travers celle-ci avoir accès à la multiculturalité des sociétés globales d'aujourd'hui. 
Universidad Pedagógica Nacional

Facultad de Humanidades

Dans ce sens, quelques institutions éducatives telles que l'université Javeriana, l'université Nacional, l'université de la Salle, l'université Libre, l'Alliance française, les centres culturels français, les lycées français et l'association colombienne de professeurs ACOLPROF ${ }^{5}$ entre autres s'engagent et contribuent concrètement à partir des initiatives de leurs programmes et de leur coopération multilatérale à préserver la langue française. Il faut aussi mentionner que l'Organisation internationale de la Francophonie, l'Agence universitaire de la Francophonie et TV5 Monde, sont parallèlement présents en faveur du français.

L’ACOLPROF (La Asociación Colombiana de profesores de francés) en particulier a été un excellent soutien du projet car ses initiatives aident à avoir un regard plus riche et vaste avec des séminaires où les stagiaires sont les premiers à raconter leurs expériences dans le projet. Nous pouvons aussi repérer l'avis de professeurs et de tous ceux qui, d'une manière ou d'une autre, y participent. L'ACOLPROF suscite le développement de la langue française, alors sa coopération au projet sera toujours essentielle. Lassociation est constamment à la recherche de moyens pour acquérir des compétences mais aussi des valeurs qui accompagnent ce processus d'enseignement du français afin de faire face aux besoins éducatifs et sociaux. Il y a donc un investissement fait par les institutions mentionnées auparavant consacrées à un seul objectif, celui d'améliorer et de chercher des solutions pour renfoncer et diffuser la langue car il y a de grandes opportunités de continuer le projet et tous les programmes éducatifs aidant à améliorer la qualité de léducation.

\section{Projet du bilinguisme au sein de l'école La Candelaria.}

Étant donné que l'école La Candelaria est une des institutions éducatives pionnières dans la réintroduction du français en Colombie, notre intérêt est centré sur la manière dont cette initiative est per-

5 ACOLPROF, Association Colombienne de professeurs de Français. www.acolprof.org çue par les participants de cette étude, à savoir : les enseignants, les élèves de l'institution et les stagiaires des derniers semestres de la licence en langues des universités, Pontificia Universidad Javeriana et Universidad Nacional de Colombia.

Pour bien commencer cette étude, nous allons aborder au départ nos perceptions initiales après avoir fait la première visite à lécole La Candelaria. Tout d'abord, nous avons remarqué que la situation à l'école est tendue car il y a une ambiance d'hyperactivité chez les élèves et du côté des professeurs on s'est rendu compte que quelques-uns n'ont pas assez de patience et qui sont parfois agressifs avec les enfants. Il ne s'agit pas de juger le comportement des acteurs de cette étude car les circonstances et les conditions ne sont pas les plus favorables au niveau social, économique et culturel. Cependant nous avons remarqué avec la réintroduction du français qu'il existe de fortes possibilités de changer d'une certaine façon une partie de cette réalité négative parce qu'il y a une motivation réelle des différents agents qui font partie de ce projet: des professeurs, des étudiants, des stagiaires et des élèves. Les institutions éducatives telles que les universités jouent un rôle essentiel permettant la circulation des stagiaires vers l'école pour promouvoir cette initiative, qui est par ailleurs nécessaire pour l'accomplissement $\mathrm{du}$ projet. Cette interaction permet de faire une analyse plus concrète de ce qui se passe à l'intérieur du programme car le stagiaire a un contact direct avec tous les acteurs du processus et ce sont eux qui peuvent témoigner grâce à leur travail. Ce travail fait par les stagiaires est une démarche éducative qui va au-delà du simple fait d'enseigner la langue française comme instrument linguistique, car leur travail a aussi une fonction sociale.

Notre premier contact avec les stagiaires a été émouvant car nous avons vu leur engagement envers le travail qu'ils font avec les élèves même si ces derniers sont des enfants rebelles qui ne possèdent pas tous les outils nécessaires pour leur apprentissage et cela rend difficile le développement du cours. Néanmoins le travail des stagiaires fait que tous ces inconvénients deviennent moins importants. 
Une autre problématique observée dans la salle de classe est l'hétérogénéité dans les niveaux deuxième, troisième, quatrième et cinquième de lécole primaire. Il y a un décalage des âges entre les 9 et les 15 ans, ce qui provoque des différences remarquables dans le parcours éducatif. Les cours étant si divers montrent notamment une inégalité dans le processus d'apprentissage chez les élèves. Carlos Castellanos $^{6}$, a fait une remarque par rapport à cette réalité, « décalage d'âge ». Il pense qu'il est important que dans son école :

«Tous les enfants doivent avoir la possibilité d'accès à l'éducation. Selon lui, il n'y a pas un argument valable pour ne pas accueillir des enfants même si leur âge ne correspond pas au niveau attendu. D’après lui, c'est un défi qu'il faut relever, il faudra aller au-delà des problématiques que cela puisse créer».

Nous pouvons pourtant dire que pour ces enfants toute évolution au niveau éducatif devient compliquée, puisque tous les problèmes présentés auparavant sont difficiles à gérer, et nous nous rendons compte que ces difficultés sont aussi produites par leur manque de possibilités et elles ont un impact négatif sur tous les niveaux de la société. Nous nous permettons de le dire, parce que la société nous concerne tous et nous savons que construire une vraie démocratie nécessite une éducation de qualité.

Il est important de former des citoyens capables de réfléchir, de critiquer, de réformer la société et ainsi de lutter contre les inégalités, entre autres. Léducation a un ingrédient très important pour construire une société et elle est le meilleur investissement qu'un Etat peut faire pour son pays et l'accès à l'éducation doit être à notre avis incontestablement un droit pour tous et surtout elle doit être accessible à tous. Cette école offre aux élèves la possibilité d'avoir accès à léducation, nonobstant, il n'y a pas assez doutils pour garantir une bonne éducation. Nous pouvons constater cette affirmation

6 Castellanos, C. Principal de l'École la Candelaria. D'après le discours fait à la I ère rencontre nationale des étudiants universitaires de français (Panorama du français dans l'université colombienne: langue, culture et formation professionnelle. Universidad Nacional de colombia. Septembre 2011 en nous servant de l'information que M. Duque ${ }^{7}$ nous a donnée.

"Quizás debo mencionar también la falta de recursos, lo cual de alguna manera es una lástima, puesto que unas simples pantallas LCD y unos micrófonos que funcionen, una fotocopiadora a color, y cosas como esas nos ayudarían enormemente a hacer clases más interesantes y a aprovechar la gran disponibilidad de textos y documentos (en el sentido amplio)".

Pourtant, la réintroduction du français au système scolaire permet un regard différent, le français ouvre un espace de réflexion pour tous les acteurs impliqués au projet. La langue française aide d'un côté, à interpréter le monde d'une manière différente parce qu'elle éveille chez les enfants un esprit créatif et innovateur pour pouvoir exprimer leurs sentiments et d'un autre côté, permet de reconnaître lautre comme un être humain.

En ce qui concerne l'importance de l'enseignement du français à l'école La Candelaria, M. Duque a dit:

"Esperamos ofrecer más alternativas para mejorar la calidad y la pertinencia de la educación de nuestros niños, niñas y jóvenes. Hemos tomado como punto de partida la consideración de que las lenguas son mucho más que códigos; ellas son un legado de la humanidad, que debemos proteger y promover, son "maneras" de interpretar el mundo, indisociables de las culturas que las originaron. En segundo lugar, nos interesan los aportes a nivel de la "flexibilidad cognitiva" (Bain, 1974; Cummis, 1976; Tunmer y Myhill; 1984) propios del estudio de lenguas, y la manera en que estas habilidades facilitan la comprensión de la diferencia, de la alteridad, con el propósito último de luchar contra la violencia que se genera en la intolerancia. Así mismo, reconocemos las ventajas que supone para una persona el manejo de "lenguas de prestigio", desde los puntos de vista cultural, de acceso a la información, de estudio y de trabajo".

7 Duque, J. Professeur de françaisà l'école la Candelaria et conseiller du programme des écoles bilingues du Secrétariat du ministère de l'éducation. 
Universidad Pedagógica Nacional

Facultad de Humanidades

Nous savons très bien que l'enseignement du français est une bonne option sur le parcours scolaire. Nous pouvons constater cette affirmation en nous servant des propos de quelques professeurs qui décrivent de manière positive la réintroduction $\mathrm{du}$ français dans lécole :

- "Hay la continuidad en el trabajo que había comenzado y no se debe dejar terminar".

- "Existe la posibilidad para que los estudiantes aprendan otra lengua".

- "Se ve una diferencia con el francés en el colegio porque los niños saben realizar con gusto algunas actividades como: saludos, preguntar el nombre, orientarse, colores, números, representaciones terrestres, relieve, dar gracias etc.".

- "Se ha permitido el conocimiento a una lengua y a una cultura diferente".

- "Los niños están animados para aprender y el aprendizaje de este idioma les resulta innovador a todos".

- "Los estudiantes hablan y cantan en francés".

M. Duque affirme que les enfants répondent de manière positive à ce qui est proposé par le projet. En plus, ils sont engagés et motivés par cette expérience et ils montrent une curiosité permanente et une envie de travailler constamment. De même, il y a un intérêt et un compromis de la part des quelques enseignants.

"Las respuestas de los niños ante las acciones del programa. Es decir, he sido sorprendido por el interés de los niños en el aprendizaje de lenguas, por la curiosidad natural ante las expresiones de otras culturas, por la rápida adquisición de aspectos tales como la prosodia, la pronunciación, cuando estos se trabajan de manera adecuada. Así mismo, puedo mencionar que algunos de los profesores $y$ directivos docentes tienen un tal grado de compromiso y de entrega para con sus estudiantes".

Comme M. Duque a dit les enfants de lécole vivent à travers le projet une autre réalité. Il faut dire que nous avons trouvé que cette expérience a de nombreux aspects positifs chez les enfants de lécole la Candelaria car ils ont envie d'aller plus loin au niveau professionnel et ils sont incités au développement de la créativité et de la curiosité. Cette dernière serait donc importante à maintenir tout au long de tous les parcours éducatifs selon le penseur anglais, J. Locke, qui affirme :

"Il faut stimuler la curiosité des sujets et la conserver constamment active : La curiosité des enfants n'est que le désir de connaître. Elle mérite donc d'être encouragée, non seulement comme un excellent symptôme, mais comme le grand instrument dont la nature se sert pour remédier à notre ignorance natives. "

La vision du monde des enfants grandit et ils reflètent tout ce que nous venons de mentionner d'après les enquêtes auxquelles ils ont répondu :

- “cuando yo sea más grande será más fácil tener una profesión y un trabajo"

- " puedo aprender otro idioma"

- “ "es bueno en mi vida porque en mi futuro puedo hacer más cosas"

- " "es un proyecto muy interesante que me sirve para el futuro"

- "Aprendo cosas que no sabía"

- "puedo ser un universitario"

- "puedo viajar"

- "Me ayuda mucho para estudiar y para salir adelante"

- "Me siento muy bien porque en otros lados no lo hacen"

- "Me gusta mucho aprender francés porque es divertido"

Lorsqu'ils sont immergés dans un contexte où la langue française est utilisée, ils apprennent aussi en s'amusant, et le fait de s'amuser est un facteur

8 Locke, J. (1693). Quelques pensées sur l'éducation, (Trad. Compayré G.), Hachette, 1889. Disponible sur: http://www.polemia.com/pdf/ Montaigne.pdf. Consulté le 07.05.2012 
important pour le développent, l'épanouissement chez l'enfant dans leur processus éducatif.

J. Locke dit qu'il faut apprendre ['apprenant] sans qu'il y voie autre chose qu'un divertissement» (1693, ibid.). » Pour cet écrivain il faudrait que l'éducation soit un instrument de joie lequel pourra faire de meilleurs individus capables d'offrir tout leur potentiel pour l'apprentissage. Les langues permettent également une ouverture au monde, les élèves se préparent à affronter un monde complexe comme celui-ci d'aujourd'hui. Avec l'enseignement de toutes les langues nous contemplons qu'elles sont inscrites dans des cadres plurilingues et pluriculturels, donnant aussi la possibilité de reconnaitre qu'il existe un « autre » avec ses différences, c'est-à-dire que les didactiques des langues vont plus loin qu'un simple enseignement.

Tout de même, malgré les avantages présentés ci-dessus, il y a des professeurs qui se sentent exclus parce qu'ils pensent que leurs opinions ne sont pas prises en considération :

«Personalmente me siento fuera del proyecto porque no cuentan con la opinión directa de los maestros, son decisiones verticales ».

D’autre part, M. Duque affirme que:

"Para algunos docentes esto simplemente significa "más trabajo" por el mismo salario, así que su participación se ve siempre condicionada a "iyo qué gano con esto?"; pero bueno, debemos tratar de entenderlos, esto obedece frecuentemente a un cierto miedo al cambio." Cela montre une attitude négative de la part de quelques professeurs parce que ce projet implique, comment ils le disent " plus de travail pour les mêmes revenus".

Nous croyons que les institutions impliquées dans le projet de réintroduction du français font des efforts pour trouver les moyens d'améliorer le processus pédagogique, mais il faudra réviser certains aspects qui ne permettent pas un bon développement, par exemple il n'y a pas de motivation ni d'intérêt de la part des professeurs pour bien s'intégrer à ce projet de bilinguisme. Nous venons de mentionner un des aspects, celui de l'attitude négative de la part de certains professeurs mais il est nécessaire de dire qu'il existe aussi d'autres aspects tels que : le manque de continuité de l'enseignement de la langue française à tous les niveaux, car il y a des étudiants qui commencent à apprendre la langue française et finissent un niveau mais il n'y a pas une garantie pour qu'ils puissent continuer avec les niveaux suivants. Les professeurs manifestent que ce serait bien d'instaurer le projet à tous les niveaux:

"el proyecto no es extensivo para todos los cursos"

" el proyecto no se ha implementado por completo

en el colegio » "Me gustaría ver el proyecto aplicado a todo el colegio".

D'autre part, nous savons que le Ministère d'Éducation a signé récemment des conventions avec l'Université Javeriana, l'Université Nationale, L'Université de la Salle, L'Université Libre et l'Alliance Française, afin de garantir aux futurs enseignants une bonne formation en langue française. Certains professeurs se forment avec une bonne attitude afin de maitriser la langue pour pouvoir l'enseigner mais il y a aussi des professeurs qui ne montrent pas d'intérêt pour cette formation car selon eux il y a toujours des inconvénients: les horaires de la formation ne leur conviennent pas, l'endroit de la formation est trop loin, ou ils ne veulent pas se former.

\section{Pourquoi ce projet de la réintroduction du français à l'école La Candelaria ?}

Nous voulons mentionner que lécole La Candelaria est la seule école publique qui fait partie de ce projet de bilinguisme en français. Voici les arguments qui nous montrent son début selon M. Duque:

"La reintroducción del francés en colegios públicos del Distrito, evento que se realizó bajo el nombre de "Aprendamos otras lenguas en el Colegio" y que se lanzó en las instalaciones del Colegio La Candelaria (Asistieron, además del Embajador y el Secretario, el Alcalde Mayor, representantes de universidades, del Liceo Francés, de la Alianza, etc.). El Colegio inicia entonces este proyecto en el "ojo del huracán", dado que es el único del programa bilingüe que eligió el 
Universidad Pedagógica Nacional

Facultad de Humanidades

francés, dada su ubicación geográfica estratégica y su énfasis en turismo y patrimonio. El acompañamiento para la transformación curricular se inicia en el marco de un contrato con la UN, con la colaboración e interventoría del Equipo de la SED; la implementación de la propuesta para el Ciclo 1 inicia en febrero de $2010 »$.

Lécole La Candelaria est située dans le quartier La Candelaria et ce quartier est considéré comme patrimoine culturel de la nation. Grâce à son emplacement il est l'un des quartiers les plus riches culturellement, ce qui fait qu'il soit très visité par les touristes. L'école devient donc, une cible dans le déroulement du programme de bilinguisme dans léducation, car c'est la seule école qui a choisi la langue française comme deuxième langue. À partir de cela, l'intérêt d'améliorer les conditions de travail des enseignants de l'école et les efforts qui se font pour rendre le projet plus efficace, pour pouvoir ainsi combler les attentes des professeurs intéressés au projet et encourager les autres professeurs qui sont plus sceptiques d'y rentrer et d'y croire.

Ceux qui ont une vision vaste du projet et qui ont une perception confiante dans l'accomplissement des tâches proposées exposent leur avis dans les affirmations suivantes :

- “La experiencia del proyecto ha sido llamativa e interesante, sobre todo porque para los estudiantes resulta innovador: ¡Todos quieren aprender francés!"

- "Posibilidad de aprender otro idioma para los estudiantes".

- “La enseñanza del francés en el colegio permite que los niños realicen con gusto algunas actividades".

- "Les ha permitido el conocimiento de otra cultura".

- "Se ve la diferencia de los niños que están dentro del proyecto porque están animados para aprender".

Pour un meilleur apprentissage il est important de souligner que la relation qu'il existe entre l'apprenant et l'enseignant est fondamentale pour le dérou- lement de la classe. Le premier contact de ces deux acteurs dans le milieu éducatif signe l'efficacité de léducation et en même temps sa qualité. Le but du projet est de proposer une meilleure éducation et satisfaire les besoins de lécole en utilisant la langue française comme instrument. Également il faudrait offrir aux enseignants des outils essentiels pour qu'ils puissent mener à bien une meilleure action enseignante auprès des enfants. L'Unesco ${ }^{9}$, dans le rapport 2005 Education pour tous - L'Exigence de qualité, nous indique la réflexion suivante :

"Un solide corpus de données fournit des indications sur ce qui fait que les écoles sont efficaces. Il met l'accent sur la dynamique du processus d'enseignement et d'apprentissage : comment enseignants et élèves interagissent dans la classe et comment ils utilisent au mieux les matériels pédagogiques. Les politiques visant à améliorer l’apprentissage doivent être centrées sur les aspects suivants. Enseignants. Ne serait-ce que pour réaliser l'EPU, il faut des enseignants plus nombreux et mieux formés. Les pays qui satisfont à des normes de haut niveau en matière d'apprentissage nont jamais cessé d'investir dans la profession enseignante. Cependant, dans beaucoup de pays, les rémunérations des enseignants se sont dégradées par rapport à celles d'autres professions au cours des deux dernières décennies, et elles sont souvent trop faibles pour permettre un niveau de vie décent. Les modèles de formation des enseignants devraient être revus dans nombre de pays afin de renforcer la formation initiale et continue dans le cadre de lécole au lieu de sappuyer sur la longue formation initiale traditionnellement dispensée dans les établissements de formation. "

Tout indique qu'il y a des efforts pour faire monter le niveau des enfants même si les difficultés restent parfois difficiles à gérer. Les apprenants peuvent aussi développer leur processus cognitif, objectif primordial de tous les systèmes éducatifs. Les enfants évoluent non seulement dans l'aspect créatif mais aussi dans l'aspect affectif.

9 UNESCO Rapport 2005 Education pour tous - L'Exigence de qualité. http://unesdoc.unesco.org/images/0013/001373/137334f.pdf. Consulté le 15.05 .2012 
Un système éducatif Intégral a besoin d'un enseignant avec les caractéristiques suivantes selon 1'Unesco ${ }^{10}$ :

- Aptitude

- Persévérance

- Disposition à la scolarité

- Connaissances préalables

- Obstacles à l'apprentissage

D’autre part, les professeurs de l'école sont accompagnés des stagiaires (des étudiants des derniers semestres des universités). Ces stagiaires font un travail très important dans ce processus, ils doivent présenter des fiches de préparation de cours et ils doivent rendre des rapports de stages à leurs universités, alors il s'agit d'un travail sérieux où ils sont engagés à faire de leur mieux pour que les résultats soient positifs. Avec nos visites et nos entretiens avec quelques stagiaires nous avons remarqué que leur fonction encourage les élèves et à quelques professeurs à relever ce défi, de même nous avons appris ce qu'ils pensent et leur position qui nous semble très pertinente car ils font partie des acteurs fondamentaux dans le projet. Voici quelques réflexions où ils manifestent leurs points de vue :

- "Notre rôle a un effet très relevant dans ce projet. Nous sommes ceux qui sont en train d'enseigner le français et de cette manière, nous sommes ceux qui peuvent contribuer à transmettre un type d'éducation bilingue. $D$ 'ailleurs, les stagiaires font des recherches qui peuvent améliorer le déroulement de ce projet. Par exemple, je suis en train de faire mon mémoire sur la création d'un matériel dans le but d'enseigner l'histoire-géo en français ».

- «Les stagiaires contribuent à l'inclusion des nouvelles méthodologies pour l'enseignement de langues. D'ailleurs, l'on a une vision moins traditionnelle de la langue et c'est pour cela que notre labour aide à motiver les étudiants pour apprendre français ».
Les stagiaires dans l'école La Candelaria s'affrontent à plusieurs contrariétés parce que les conditions ne sont pas toujours les meilleures, ils pensent qu'il y a un manque de ressources et d'intégration entre les professeurs et les stagiaires. Nous estimons que malgré le travail fait au jour le jour, il mériterait de faire encore plus d'efforts et de continuer la recherche des conditions convenables pour le projet. Ils pensent que :

- «Je pense qu'il y a beaucoup des efforts qui a fait "la secrétariat de éducation » et l'ambassade de France avec ce projet. Cependant, il manque beaucoup des éléments sur lesquels le gouvernement doit travailler ».

- «En première lieu, il y a une pénurie des ressources pédagogiques dans le deuxième cycle d'application $\left(2^{\circ}-5^{\circ}\right.$ primaria $)$ de ce projet. Cela veut dire qu'il manque un programme complet pour l'enseignement du français comme langue étrangère et dans les disciplines non linguistiques (histoire-géographie). De plus, il n'y a qu'un livre (Tatou), mais ce matériel n'est pas approprié pour tous les étudiants ».

- "En deuxième lieu, il manque une intégration entre les professeurs et les stagiaires pour organiser la gestion du groupe et l'enseignement des contenus. Si on tient en compte que la population de cet école es très hétérogène, il est vraiment nécessaire que le professeur s'involucre dans le cours de français et qu'il aide avec le control des situations violents dans la salle de classe».

- " Je considère que ce projet a des bonnes intentions pour l'éducation bilingue en Colombie. Cependant, il manque encore des éléments pour qu'il puisse marcher correctement. Par exemple, L'école La Candelaria n’a pas des professionnels qui connaissaient les contenus et la langue étrangère. C’est pour cela que les stagiaires de différentes universités peuvent ou doivent essayer d'enseigner disciplines non linguistiques (le cas des sta- 
Universidad Pedagógica Nacional

Facultad de Humanidades

giaires de la Javeriana) sans avoir un livre ou une méthodologie qui représente une aide pour eux ».

Nous pensons que ce projet est une bonne initiative, le fait d'apporter de nouvelles ressources qui puissent aider à améliorer l'éducation va être toujours ouvert à des propositions et aussi à des possibilités pour l'avenir de l'éducation.

\section{Conclusions}

Létude de la langue française dans le processus de la réintroduction du français en Colombie révèle la complexité présente dans tous les contextes concernés par cet apprentissage. Il faut croire, promouvoir et éveiller la curiosité chez lélève car nous avons vu à travers cette étude la valeur de la langue comme un mécanisme qui permet l'apprentissage de la langue non seulement comme un instrument linguistique mais aussi comme un instrument social.

Le contexte de lécole la Candelaria est un reflet de notre société et nous avons remarqué que cette société a besoin d'une éducation de qualité, laquelle nest pas totalement donnée en raison des inconvénients et les problématiques au niveau social, culturel et économique. Nous avons vu que s'il n'y a pas une éducation de qualité les individus ne peuvent pas développer des capacités pour affronter leur place au sein de la société d'aujourd'hui.

Le projet de la réintroduction du français en Colombie cherche des moyens pour que les élèves aient de bons résultats dans leur processus d'apprentissage. Ce parcours se caractérise par le développement de compétences et des valeurs pouvant améliorer d'une façon positive leur rôle dans la société car nous pouvons voir qu'il y a beaucoup d'efforts pour développer une éducation de base et que les élèves acquièrent des connaissances qui les aident à faire face au monde tel qu'il est aujourd'hui.

D'après les perceptions de tous les participants actifs dans le projet de bilinguisme de la réintroduction du français en Colombie, nous avons aperçu qu'il y a des intérêts pour atteindre les objectifs proposés dans ce projet malgré le manque des res- sources à tous niveaux. Il est évident qu'il y a des résultats positifs parce que la plupart des acteurs sont à faveur du projet et nous voyons leur enthousiasme. Ce dernier est un facteur important car s'il y a une bonne disposition de la part de tous, le projet pourra se fortifier.

\section{Bibliographie}

Breton A. (1978) «Le bilinguisme : une approche économique ». Institut C.D. Howe, Montréal-Canada.

Brunot, F. (1979) « Histoire de la langue française des origines à nos jours », Armand Colin, Paris.

Roy, J. L. (2008) « Quel avenir pour la langue française?: Francophonie et concurrence culturelle au XXIe siècle ». Montréal, Hurtubise HMH. 268 p. (ISBN 9782896471522). [Page Web]: http://www.cerium. ca/Quel-avenir-pour-la-langue, 7779

Calvet, L. J. (2007) "Approche sociolinguistique de l'avenir du français dans le monde ", Hérodote 3 ( $\mathrm{n}^{\circ}$ 126), p. 153-160. (ISBN 0338-487X). [Page Web]: http://www.apprendrellangue.com/ langue-minoritaire-mondialisation/

Mothe, J.C. (1977) «Evaluation de l'enseignement du français en Colombie ». Rapport d'une mission effectuée à Bogotá, Cali, Popayán, Cartagena et Medellín du 5 au 11 décembre 1977, B.E.L.C., Miméo, Paris.

Locke, J. (1693). “Quelques pensées sur l'éducation”. (Trad. Compayré G.), Hachette, 1889. [Page Web]: http://www.polemia.com/pdf/Montaigne.pdf.

Montaigne, M. (1595). “OEuvres completes”. Éd. Albert Thibaudet, Maurice Rat, Paris: Gallimard, 1962.

Montaigne, M. (1595). "L'éducation des enfants, Du pédantisme et de l'institution des enfants" (Chapitres 25 et 26 du Livre I des Essais), (prés. Par Pinganaud C.), Arléa.

UNESCO. "Rapport 2005 Education pour tous L'Exigence de qualité ». [Page Web]: http://unesdoc.unesco.org/images/0013/001373/137334f.pdf.

Hargreaves, A. (2003) «Enseñar en la sociedad del conocimiento »ISBN: 978-84-8063-578-3. Ediciones octaedro, s.l.

Mejia, M. R.__ "Educacion Popular hoy en Tiempos de Globalizacion ». Aurora ediciones. ISBN 9789589136188 